\title{
CENP-H regulates the cell growth of human hepatocellular carcinoma cells through the mitochondrial apoptotic pathway
}

\author{
GUIFANG LU ${ }^{1}$, HELEI HOU ${ }^{2}$, XINLAN LU ${ }^{1}$, XIQUAN KE $^{1}$, XIN WANG $^{1}$, DAN ZHANG $^{1}$,

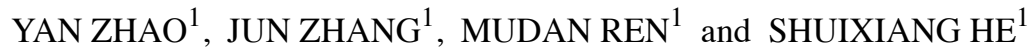 \\ ${ }^{1}$ Department of Gastroenterology, The First Affiliated Hospital of Xi'an Jiaotong University, Xi'an, Shaanxi 710061;
${ }^{2}$ Department of Medical Oncology, The Affiliated Hospital of Qingdao University, Qingdao, Shandong 266003, P.R. China
}

Received August 23, 2016; Accepted November 14, 2016

DOI: $10.3892 /$ or.2017.5602

\begin{abstract}
The genomic alterations of hepatocellular carcinoma (HCC) are still unclear. Centromere protein-H (CENP-H) has been shown to be associated with many solid tumors. Our previous study found that CENP-H was upregulated in $\mathrm{HCC}$ and was related to patient prognosis. However, the biological functions of CENP-H in HCC and the possible underlying mechanisms have not been well elucidated. In the present study, we demonstrated that CENP-H knockdown inhibited the proliferation of Hep3B cells and decreased colony formation ability of single cells in vitro. Furthermore, CENP-H knockdown induced Hep3B cell apoptosis, and apoptotic bodies were observed using transmission electron microscopy. The protein expression of cleaved caspase-3 was upregulated in Hep3B cells after CENP-H knockdown. Additionally, a Bax/Bcl-2 ratio imbalance with a significant increase of Bax and a substantial decrease of Bcl-2 at both the mRNA and protein levels were determined in this study. In an animal experiment, CENP-H knockdown blocked the growth of Hep3B subcutaneous xenografts. Immunohistochemistry revealed that the protein expression of cleaved caspase- 3 and Bax was increased, whereas the protein expression of $\mathrm{Bcl}-2$ and $\mathrm{Ki}-67$ was decreased in subcutaneous xenografts of the CENP-H-knockdown group. In summary, CENP-H may be involved in cell proliferation and apoptosis of HCC cells through the mitochondrial apoptotic pathway. Combined with previous studies, the data provide a new perspective on HCC development and progression.
\end{abstract}

\section{Introduction}

Hepatocellular carcinoma (HCC) is one of the most common malignancies worldwide, and $50 \%$ of all newly diagnosed $\mathrm{HCC}$

Correspondence to: Dr Mudan Ren or Professor Shuixiang He, Department of Gastroenterology, The First Affiliated Hospital of Xi'an Jiaotong University, 277 Yanta West Road, Xi'an, Shaanxi 710061, P.R. China

E-mail: 50490185@qq.com

E-mail: hesx123@126.com

Key words: CENP-H, hepatocellular carcinoma cells, proliferation, apoptosis cases and deaths worlwide are estimated to occur in China every year $(1,2)$. In recent years, the incidence rate of HCC in the US has increased, and the 5-year survival rate after cancer diagnosis is only $18 \%$ (3). Compared with other lethal cancers, the genomic alterations of HCC are poorly understood due to its complexity and heterogeneity (4). In depth studies on the genomic alterations may not only help to better understand the pathogenesis of HCC, but may also facilitate the development of new treatment strategies.

Chromosomal instability (CIN), a major cause of genomic instability that is thought to be critical in the development of human cancer, arises from inaccurate separation of sister chromatids in mitosis (5). Kinetochores that consist of more than 15 centromere proteins (CENPs) are vital for accurate chromosome segregation by mediating microtubule attachment and controlling the movement of chromosomes during mitosis and meiosis (6). Inappropriate expression of CENPs may be a major cause of CIN and tumorigenesis (7). Centromere protein-H (CENP-H), a fundamental component of the human active centromere complex (8), has been found to be upregulated in human colorectal cancer, and the ectopic expression of CENP-H induces aneuploidy (9). Recently, accumulating evidence revealed that the abnormal expression of CENP-H is closely associated with several cancers including esophageal $(10)$ and gastric carcinoma $(11,12)$, breast $(13)$ and uterine cervical cancer (14).

Our previous research demonstrated that CENP-H was not only overexpressed in HCC samples, but was also closely related to tumor size, histological grade and clinical stage. Moreover, it may be a novel prognostic biomarker of HCC progression (15). In the present study, we investigated the role of CENP-H in HCC cell survival. The results confirmed that CENP-H knockdown in Hep3B cells induced growth arrest and apoptosis in vitro and in vivo, and the mitochondrial apoptotic pathway may play a key role in this process.

\section{Materials and methods}

Ethics statement. All animal protocols complied with the National Institutes of Health Guide for the Care and Use of Laboratory Animals with the approval of the Institutional Animal Care and Use Committee of Xi'an Jiaotong University. 
Cell lines and cell culture. HCC cell lines Hep3B, HepG2, HHCC, SMMC7721 and MHHC97H and immortalized human liver cells L02 were purchased from the Institute of Cellular Research of the Chinese Academy of Sciences (Shanghai, China). All of the cell lines were cultured in Dulbecco's modified Eagle's medium (DMEM; Gibco, Grand Island, NY, USA) supplemented with $10 \%$ fetal bovine serum (FBS; HyClone, Logan, UT, USA) and $1 \%$ penicillin/streptomycin, and incubated at $37^{\circ} \mathrm{C}$ in a humidified $5 \% \mathrm{CO}_{2}$ atmosphere.

$R N A$ isolation and real-time $q P C R$. Total RNA was extracted with TRIzol reagent (Invitrogen, Carlsbad, CA, USA) according to the manufacturer's instructions. First-strand cDNA was synthesized from total RNA with PrimeScript $^{\circledR}$ RT Master Mix (Takara Biotechnology Co., Ltd., Dalian, China). Real-time quantitative qPCR was performed (Bio-Rad Laboratories, Hercules, CA, USA) with SYBR ${ }^{\circledR}$ Premix Ex Taq ${ }^{\mathrm{TM}}$ II (Takara Biotechnology). The cycling conditions were as follows: initial denaturation at $95^{\circ} \mathrm{C}$ for $30 \mathrm{sec}$, and then 40 cycles of denaturation at $95^{\circ} \mathrm{C}$ for $5 \mathrm{sec}$ and annealing and elongation at $60^{\circ} \mathrm{C}$ for $30 \mathrm{sec}$. Bio-Rad CFX Manager 2.1 software was used for qPCR analysis. The GAPDH housekeeping gene was used as an internal control.

The PCR primers sets were as follows: i) CENP-H forward, 5'-CAGTCTAGTGTGCTCATGGAT-3' and reverse, 5'-TCCATCTGTAGGTTTTGTCG-3'; ii) Bax forward, 5'-GGTTGTCGCCCTTTTCTACTTT-3' and reverse, 5'-GTGAGGAGGCTTGAGGAGTCT-3'; and iii) Bcl-2 forward, 5'-TCCCATCAAAACTCCTGTCTTT-3' and reverse, 5'-GCAAGTGAATGAACACCTTCTC-3'; iv) glyceraldehyde-3-phosphate dehydrogenase (GAPDH) forward, 5'-CAAGCTCATTTCCTGGTATGAC-3' and reverse, 5'-CAGTGAGGGTCTCTCTCTTCCT-3'.

Protein extraction and western blotting. Total cell protein was extracted according to a previous study (15). After denaturation, equal amounts of total protein were separated electrophoretically on $10 \%$ SDS/polyacrylamide gels (SDS-PAGE) and transferred onto polyvinylidene difluoride (PVDF) membranes (Millipore, Bedford, MA, USA) using a tank transfer apparatus (Bio-Rad Laboratories). The membranes were blocked with 5\% skim milk in Trisbuffered saline with Tween (TBS-T) for $2 \mathrm{~h}$, then incubated with primary antibodies overnight at $4{ }^{\circ} \mathrm{C}$. The following day, after the incubation with horseradish peroxidase-conjugated secondary antibodies, the blots were visualized with enhanced chemiluminescence horseradish peroxidase (HRP) substrate (Millipore) according to the manufacturer's instructions. The intensity of each band was assessed using Image Lab 4.0 (Bio-Rad). The following primary antibodies were used: anti-CENP-H (1:200; Santa Cruz Biotechnology, Inc., Santa Cruz, CA, USA), anti-Bax $(1: 1,000)$, anti-Bcl-2 (both from Epitomics, Abcam, CA, USA, 1:2,000) and anti-cleaved caspase-3 (1:1,000; Cell Signaling Technology, Beverly, MA, USA). To confirm consistent loading, mouse anti- $\beta$-actin antibody (Santa Cruz Biotechnology, Inc.) diluted 1:1,000 was used as a primary antibody. The secondary antibodies were purchased from Santa Cruz Biotechnology, Inc. and were diluted 1:5,000.
Lentiviral packaging and infection. To knock down CENP-H expression, 2 lentiviruses with different siRNA sequences were packaged using the LV-3 (pGLVH1/GFP + Puro) vector (GenePharma, Shanghai, China). The siRNA sequences were as follows: CENP-H1, 5'-TGGTTGATGCAAGTGAAGA-3'; and CENP-H2, 5'-GCTTGAGAAGAATGTTGACAT-3'. Concomitantly, the negative controls were also packaged. Puromycin (2.5 $\mu \mathrm{g} / \mathrm{ml}$; Sigma, St. Louis, MO, USA) was used to kill the uninfected Hep3B cells. Hep3B cells that were stably infected with CENP-H1 and CENP-H2 were named LV3-CENP-H1 and LV3-CENP-H2, respectively, and the negative control was named LV3-NC. Real-time PCR and western blotting were used to detect knockdown efficiency, and the virus with the highest knockdown efficiency was used in the subsequent experiments.

Proliferation assay. The 3-(4,5-dimethylthiazol-2-yl)-2,5-diphenyltetrazolium bromide (MTT) assay was performed to detect cell proliferation. Hep3B, LV3-CENP-H1 and LV3-NC cells were separately seeded into 96-well plates at a density of $2 \times 10^{3}$ cells/well (5 repeats/cell line), after culture for 0-7 days; MTT reagent (Sigma), which was freshly prepared, was added to each well. Following $4 \mathrm{~h}$ of incubation, the supernatant was discarded, and dimethyl sulfoxide (DMSO) (Sigma) was added to dissolve the crystals. All analyses were performed in triplicate. The absorbance was assessed at a test wavelength of $490 \mathrm{~nm}$ using an EnSpire ${ }^{\mathrm{TM}}$ Multilabel Reader (PerkinElmer, Waltham, MA, USA).

Colony formation assay. Hep3B, LV3-CENP-H1 and LV3-NC cells were seeded in fresh 6-well plates at a density of 500 cells/well in DMEM with 10\% FBS. After 10 days, the cells were washed with PBS, fixed in $4 \%$ paraformaldehyde for $30 \mathrm{~min}$, and stained with $0.5 \%$ crystal violet for $20 \mathrm{~min}$. Colonies were scored, and each experiment was repeated 3 times.

Cell apoptosis analysis using flow cytometry. After $72 \mathrm{~h}$ of incubation, Hep3B, LV3-CENP-H1 and LV3-NC cells were harvested, washed twice with cold PBS, diluted in $1 \mathrm{X}$ binding buffer, stained with recombinant human Annexin V-Alexa Fluor 647 and PI using Annexin V-Alexa Fluor 647 apoptosis detection kit (Beijing 4A Biotech Co., Ltd, Beijing, China) according to the manufacturer's instructions, and then analyzed using a FACSCanto $^{\mathrm{TM}}$ II Flow Cytometer (BD Biosciences, San Jose, CA, USA).

Transmission electron microscopy (TEM). After $72 \mathrm{~h}$ of incubation, Hep3B, LV3-CENP-H1 and LV3-NC cells were harvested. The collected cells were first fixed in $2.5 \%$ glutaraldehyde for $2 \mathrm{~h}$ at $4^{\circ} \mathrm{C}$, then rinsed in $0.1 \mathrm{M}$ phosphate buffer (PB) (pH 7.4), and post-fixed in 1\% osmium tetraoxide for $2 \mathrm{~h}$. After being washed in PB, the cells were progressively dehydrated in a graded series of ethanol, and then cleared in QY-1 (Nissin EM, Tokyo, Japan). Then, embedding in epoxy resin 812 and sectioning were performed, and the ultrathin sections (60-nm thickness) were stained with both uranyl acetate and lead citrate. Finally, cellular ultramicroscopic structures were examined by TEM (Hitachi, Tokyo, Japan). 
Tumorigenicity in nude mice. Male 4-6 week-old BALB/c nude mice were purchased from the Experimental Animal Center of Shanghai, and fed in specific pathogen-free (SPF) animal rooms with constant temperature and humidity at the Centre of Laboratory Animals of The Medical College of Xi'an Jiaotong University. After 4 days of adaptive feeding with an autoclaved chow diet with water, the mice were randomly divided into 2 groups (4 mice/group), and $2 \times 10^{6}$ LV3-CENP-H/LV3-NC cells were subcutaneously injected into the right thigh of each mouse. The body weights of the mice and tumor volumes were assessed every 4-5 days, and the tumor volume was calculated using the following formula: Tumor volume $=$ (tumor length $\mathrm{x}$ tumor width $\mathrm{x}$ tumor width)/2. After 4 weeks of observation of xenograft tumor formation, the mice were sacrificed, and the tumor tissues were obtained as soon as possible. After being washed with PBS, some of the tumor samples were fixed with $4 \%$ formalin and paraffin-embedded for histological studies, and others were immediately snap-frozen in liquid nitrogen and stored at $-80^{\circ} \mathrm{C}$ for long-term preservation.

Immunohistochemistry analysis. Xenograft samples were immediately fixed in $4 \%$ formalin once obtained from the mice. After paraffin-embedding, $4-\mu \mathrm{m}$ thick paraffin sections were adhered to glass slides and prepared for immunohistochemical assay using a standardized streptavidin-peroxidase (SP) method as previously reported (15). Following deparaffinization, rehydration, antigen retrieval and blockage, the slides were incubated with primary antibodies overnight at $4^{\circ} \mathrm{C}$. The following day, after secondary antibody incubation and dehydration, the slides were examined under a microscope (Olympus, Tokyo, Japan). The primary antibodies included anti-CENP-H (1:40; Santa Cruz Biotechnology, Inc.), anti-Bax, anti-Bcl-2 (1:100) (both from Epitomics), anti-cleaved caspase-3 (1:300) and anti-Ki-67 (1:200) (both from Cell Signaling Technology). The secondary antibodies were from Zhongshan Golden Bridge Biotechnology (Beijing, China).

Statistical analysis. The experimental data are presented as the mean \pm SEM and were analyzed using the two-tailed Student's t-test. $\mathrm{P}<0.05$ was considered statistically significant. Statistical analysis was performed using SPSS 19.0 statistical software (SPSS, Inc., Chicago, IL, USA).

\section{Results}

The expression of CENP-H in HCC cell lines and immortalized human liver cells. To investigate the protein and gene expression levels of CENP-H in different HCC cell lines and immortalized human liver cells, total protein and mRNA were extracted from Hep3B, HepG2, HHCC, SMMC7721, MHHC97H and L02 cells, separately. Western blotting revealed that the CENP-H protein levels in all HCC cell lines were higher than that in the L02 cells (Fig. 1A and B). Real-time PCR analysis produced similar results as the western blotting assay (Fig. 1C). These results demonstrated that CENP-H was upregulated at both the protein and mRNA levels in HCC cell lines and suggest that it may play an important role in HCC progression. Additionally, considering the high endogenous expression of CENP-H in the Hep3B cell line, the Hep3B cell line was chosen for the following knockdown experiments.
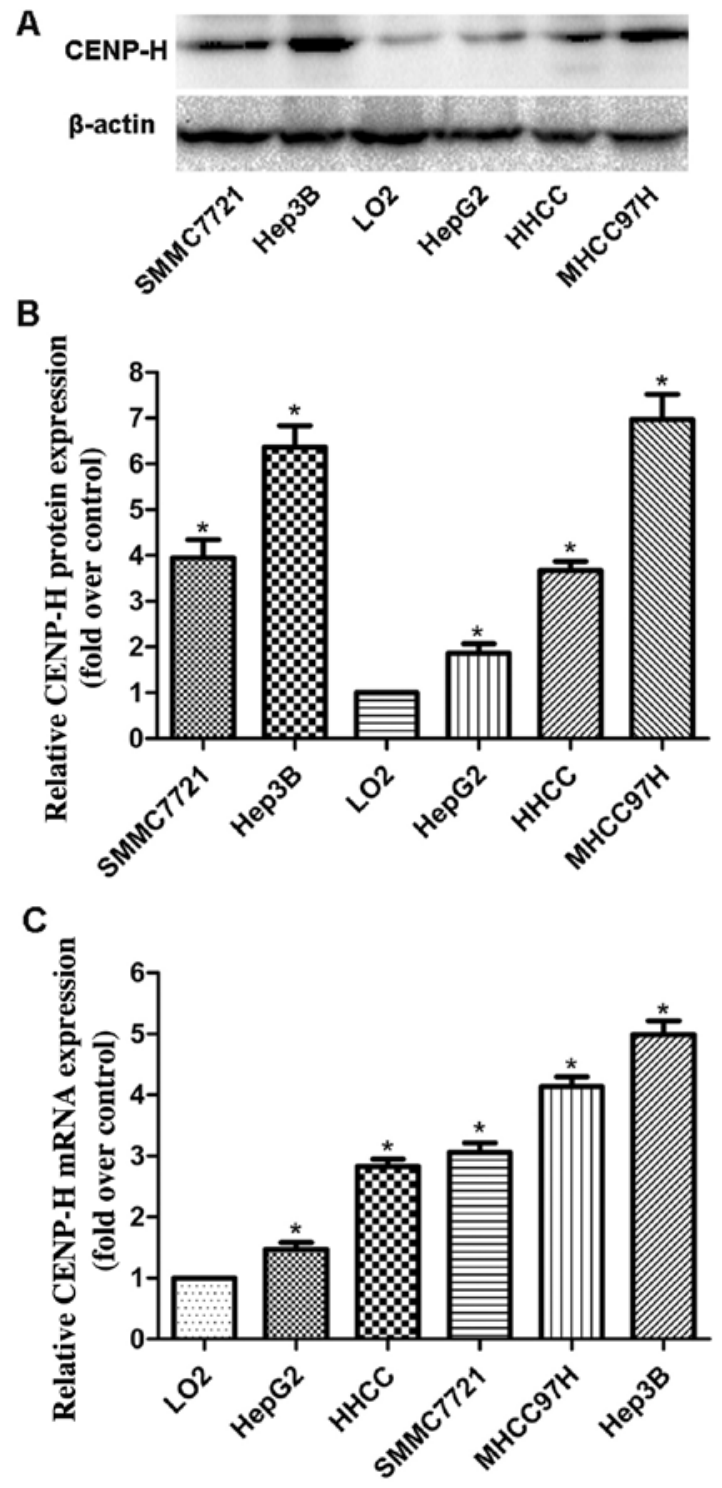

Figure 1. The protein and mRNA expression of CENP-H in HCC and immortalized liver cells. (A and B) CENP-H protein expression. (C) CENP-H mRNA relative quality. Values are expressed as the mean \pm standard error; ${ }^{*} \mathrm{P}<0.05$ vs. the L02 cells. CENP-H, centromere protein-H; HCC, hepatocellular carcinoma.

CENP-H knockdown inhibits cell proliferation in Hep3B cells. To elucidate the biological function of CENP-H in HCC cells, we packaged lentiviruses to infect Hep3B cells in order to construct stable shRNA-mediated CENP-H knockdown. After $72 \mathrm{~h}$ of incubation, the viral infection efficiency was $>90 \%$ using fluorescence microscopy (Fig. 2A). To further validate the knockdown efficiency, real-time PCR and western blotting were performed. The results confirmed that compared with the negative control, both the siRNA sequences significantly eliminated the endogenous CENP-H expression in the Hep3B cells (Fig. 2B-D). Taking knockdown efficiency into account, we chose LV3-CENP-H1 for the subsequent biological experiments.

The MTT and colony formation assays were used to detect the changes in the proliferative capacity of Hep3B after CENP-H knockdown. The MTT results revealed that LV3-CENP-H1 cells had a lower proliferative ability than 
A

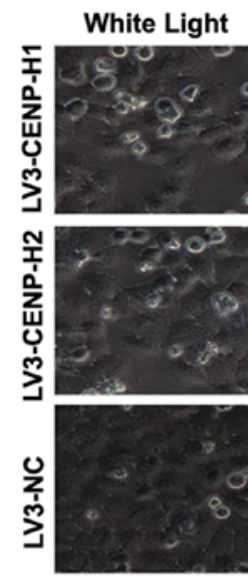

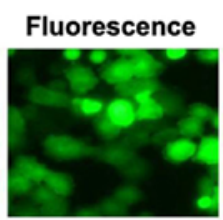

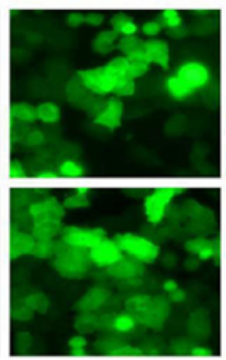

Merge

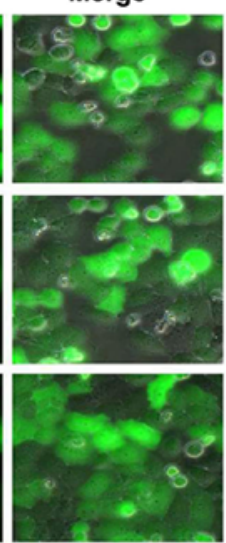

B

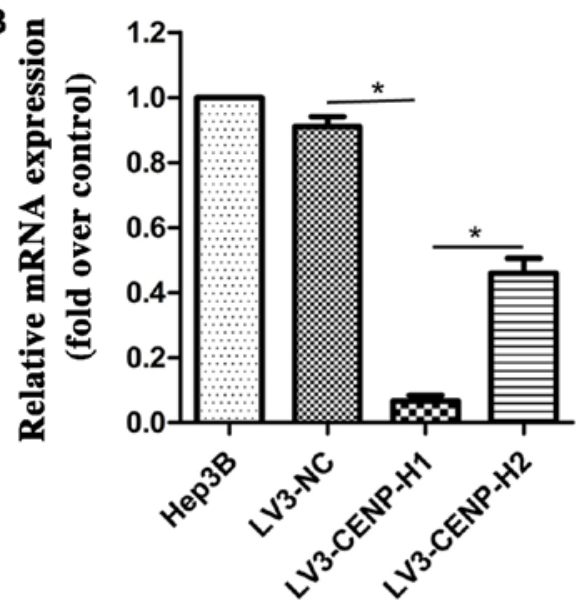

D
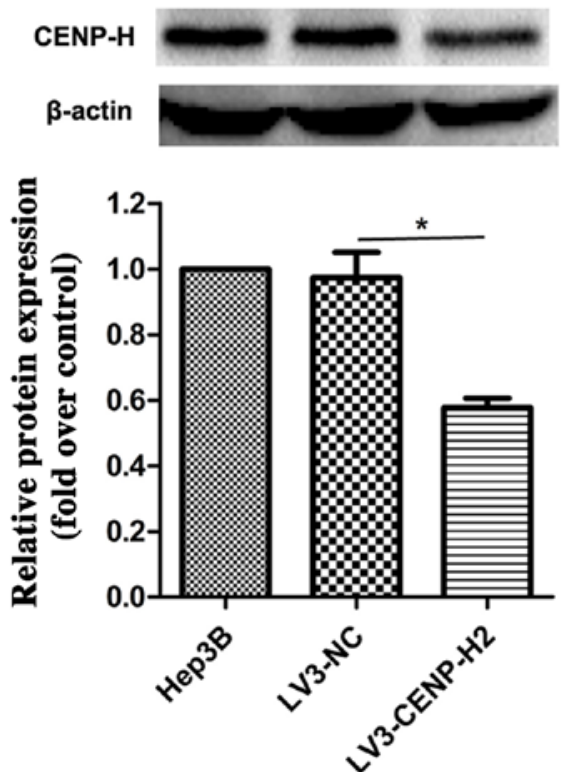

Figure 2. CENP-H knockdown efficiency. (A) Cell fluorescence after a 72-h viral infection. (B) CENP-H mRNA knockdown efficiency. (C and D) CENP-H protein knockdown efficiencies. Values are expressed as the mean \pm standard error; ${ }^{\mathrm{P}}<0.05$. CENP-H, centromere protein-H.

the Hep3B and LV3-NC cells. In addition, the proliferative abilities of the Hep3B and LV3-NC cells did not significantly differ (Fig. 3A). CENP-H knockdown suppressed the colony formation ability of the Hep3B cells (Fig. 3B). Both the MTT and colony formation assay results demonstrated that CENP-H knockdown inhibits Hep3B proliferation.

CENP-H knockdown induces apoptosis of Hep3B cells. Flow cytometric and TEM assays were used to detect cell apoptosis. The apoptotic index was increased from $3.13 \pm 0.42 \%$ in the LV3-NC cells to $19.96 \pm 0.5 \%$ in the LV3-CENP-H1 cells after CENP-H knockdown (Fig. 4A). Through TEM, we observed chromatin condensation, mitochondrial swelling and apoptotic bodies in the LV3-CENP-H1 cells (Fig. 4B). Both the flow cytometric and TEM results indicated that CENP-H knockdown induced apoptosis of the Hep3B cells. Based on western blotting, we found that cleaved caspase- 3 expression was increased in the LV3-CENP-H1 cells compared with the level noted in the Hep3B and LV3-NC cells (Fig. 5A).

Based on swollen mitochondria observed using TEM, the apoptosis-related factors involved in the mitochondrial apoptotic pathway were detected. Both protein and mRNA expression levels of Bax were increased in the LV3-CENP-H1 cells compared to these levels in the Hep3B and LV3-NC cells (Fig. 5B and D). Conversely, the protein and mRNA expression levels of Bcl-2 were downregulated after CENP-H knockdown (Fig. 5C and E).

CENP-H knockdown suppresses tumor growth in a Hep3B cell xenograft model. The Hep3B xenograft model was used to determine whether CENP-H knockdown affects tumor growth in vivo. The LV3-CENP-H1 group of animals formed much smaller tumors from 20 days after cell inoculation than the control group. After sacrifice, we confirmed that the average tumor weight in the LV3-CENP-H1 group was lighter than in the control. In addition, the average tumor volume in the LV3-CENP-H1 group was also smaller than in the control (Fig. 6). Immunohistochemistry for CENP-H confirmed the decreased staining in the LV3-CENP-H1 group of tumors compared with the LV3-NC tumors. Similarly to the in vitro experiment results, the LV3-CENP-H1 tumors exhibited increased staining for cleaved caspase-3 and 
A

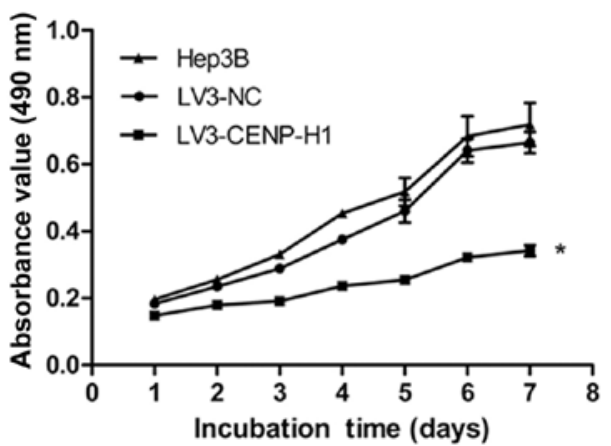

B
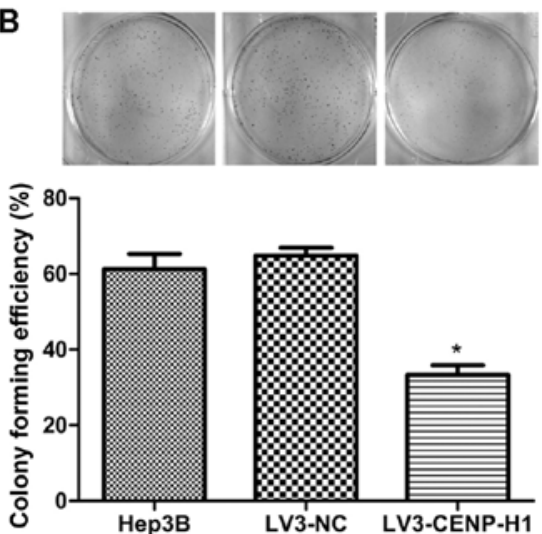

Figure 3. CENP-H knockdown inhibits the proliferation of Hep3B cells. (A) CENP-H knockdown inhibited the proliferation of Hep3B cells. (B) CENP-H knockdown decreased colony formation ability of Hep3B cells. Values are expressed as the mean \pm standard error, ${ }^{*} \mathrm{P}<0.05$ vs. the LV3-NC and Hep3B cells. CENP-H, centromere protein-H.

A

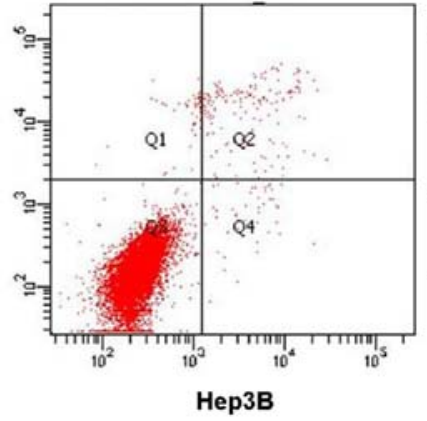

B

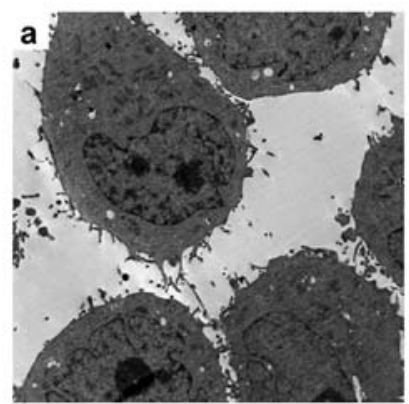

d

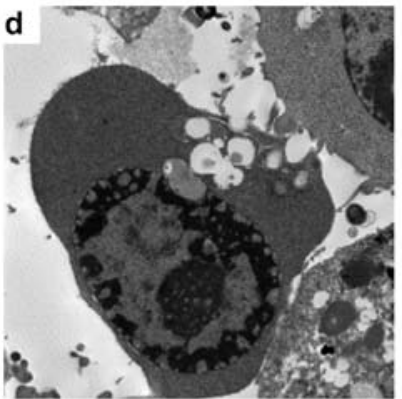

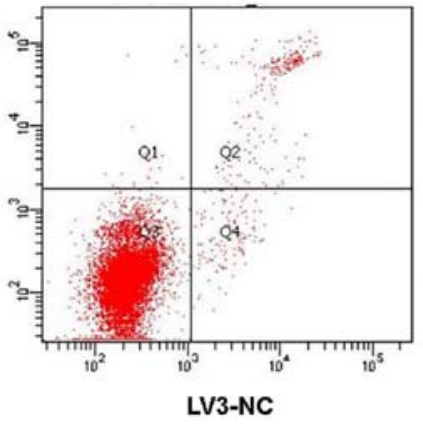
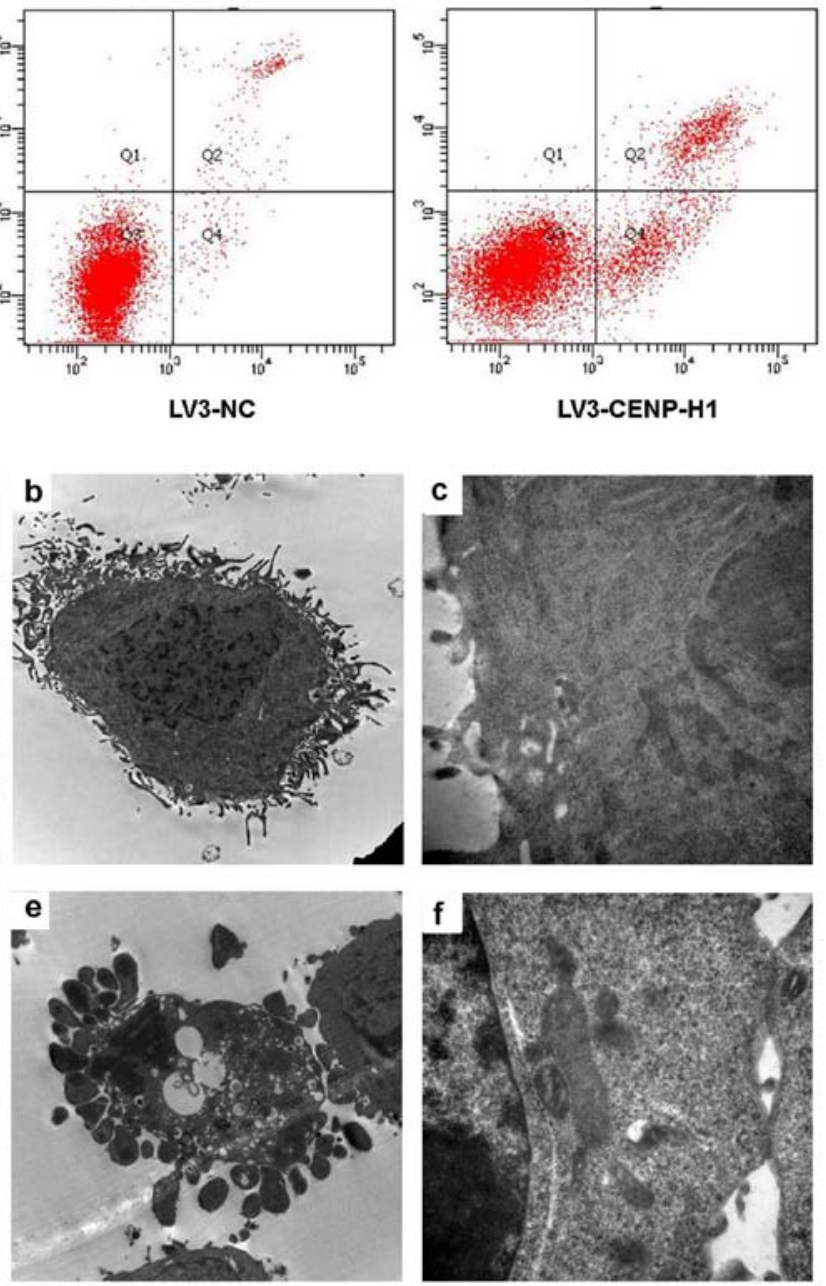

Figure 4. CENP-H knockdown induces apoptosis of Hep3B cells. (A) CENP-H knockdown induced apoptosis of Hep3B cells. (B) Representative transmission electron micrographs demonstrating the morphology and ultrastructure of CENP-H knockdown Hep3B cells. a, Magnification (x8,000), cell morphology of Hep3B cells; b, magnification (x8,000), cell morphology of LV3-NC cells; c, magnification (x40,000), cell ultrastructure in LV3-NC cells; d, magnification $(\mathrm{x} 8,000)$, chromatin margination and vacuolization in LV3-CENP-H1 cells; e, magnification (x8,000), apoptotic bodies in LV3-CENP-H1 cells; f, magnification (x40,000), obvious mitochondrial swelling in LV3-CENP-H1 cells. Values are expressed as the mean \pm standard error; ${ }^{*} \mathrm{P}<0.05$ vs. the LV3-NC and Hep3B cells. CENP-H, centromere protein-H.

Bax, compared with the LV3-NC tumors. In addition, the expression of Bcl-2 decreased in the LV3-CENP-H1 group.
Immunohistochemistry for Ki-67 was used to detect the proliferative activities of both the LV3-CENP-H1 and LV3-NC 
A
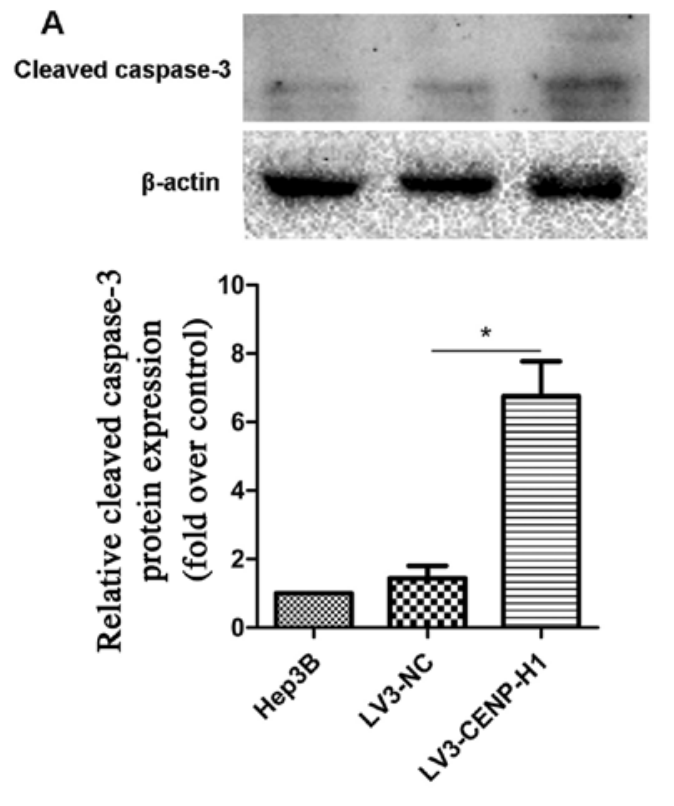

C

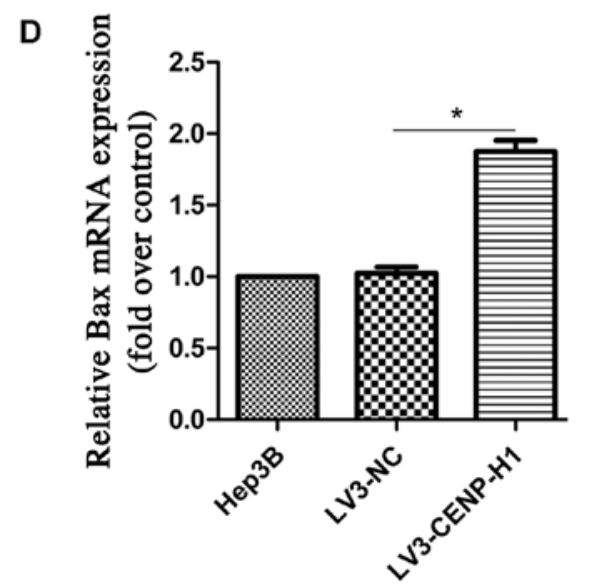

B
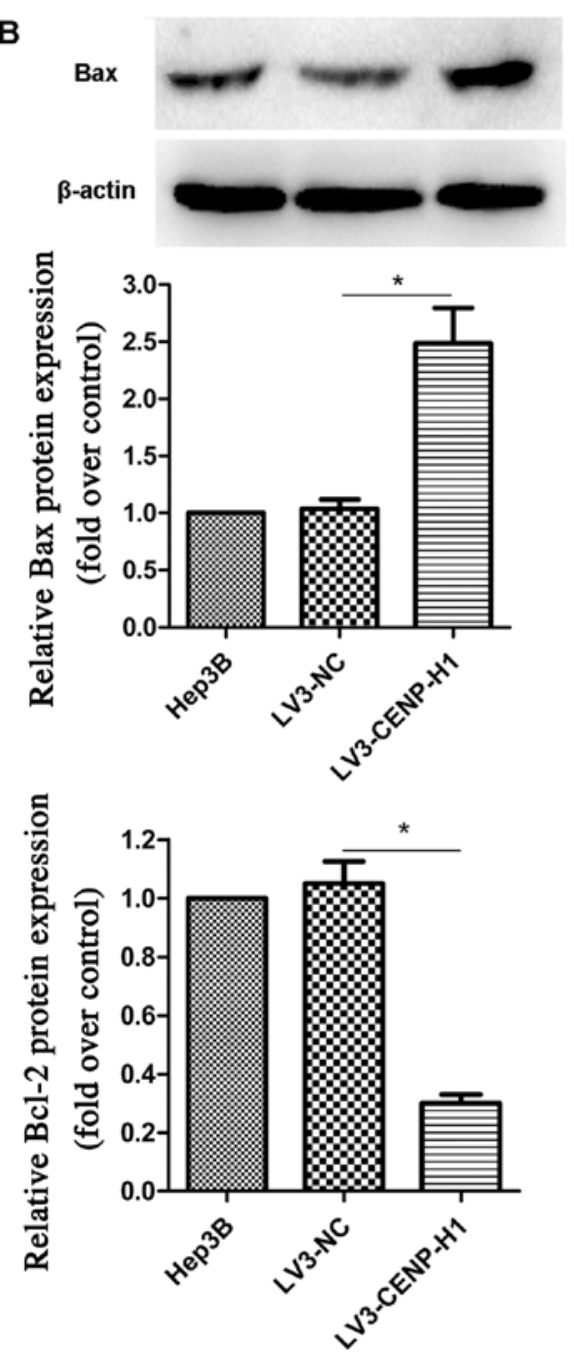

E

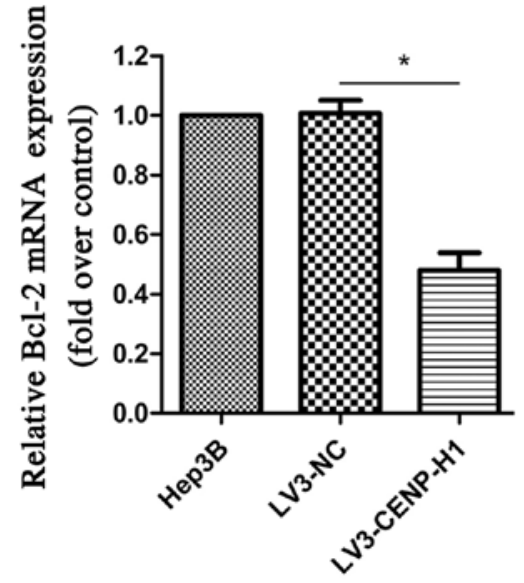

Figure 5. CENP-H knockdown induces activation of caspase-3 with upregulation of Bax expression and downregulation of Bcl-2 expression at both mRNA and protein levels. (A) CENP-H knockdown increased cleaved caspase-3 protein expression. (B) CENP-H knockdown increased Bax protein expression. (C) CENP-H knockdown decreased Bcl-2 protein expression. (D) CENP-H knockdown increased Bax mRNA expression. (E) CENP-H knockdown decreased Bcl-2 mRNA expression. Values are expressed as the mean \pm standard error; $\mathrm{P}<0.05$. CENP- $\mathrm{H}$, centromere protein- $\mathrm{H}$.

tumors. As expected, the LV3-CENP-H1 tumors had a lower Ki-67 expression than the LV3-NC tumors (Fig. 7).

\section{Discussion}

HCC is not only the sixth most common neoplasm, but is also the third leading cause of cancer-related deaths worldwide (16).
Hepatocarcinogenesis is a complex multistep process in which many genes and signaling pathways are involved. In our previous research, we found that CENP-H expression is not only upregulated in HCC tissues at both the mRNA and protein levels but is also closely related to tumor size, histological grade, and clinical stage. Additionally, CENP-H expression based on immunohistochemistry was negatively 
A
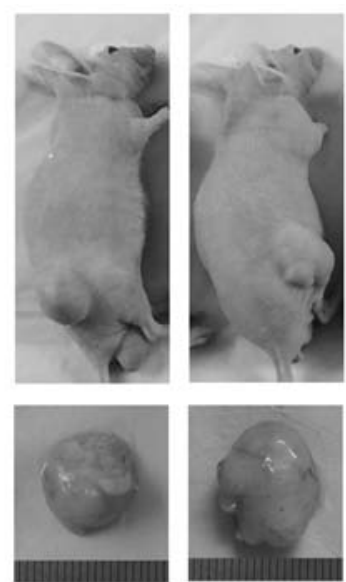

C

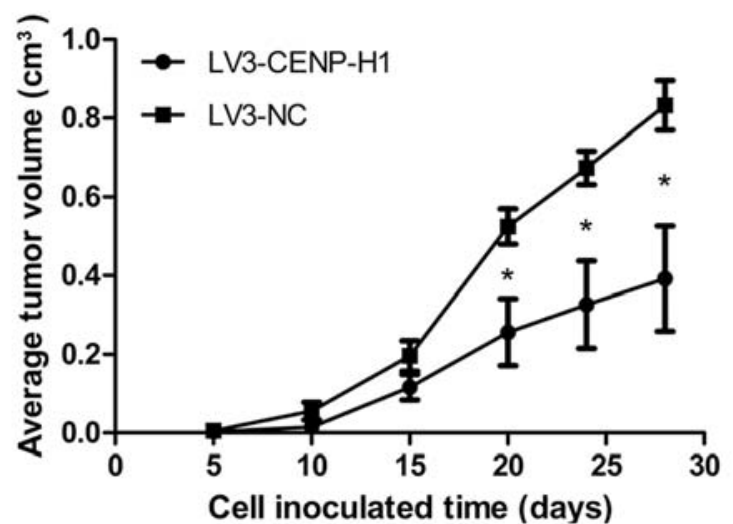

B
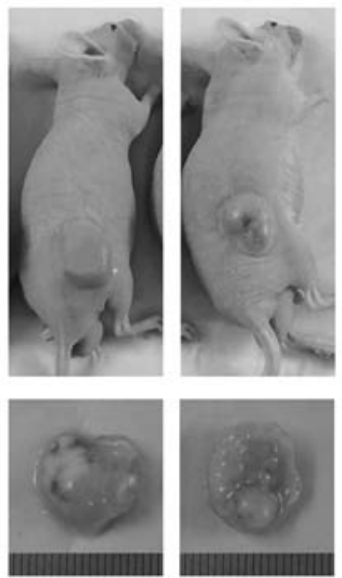

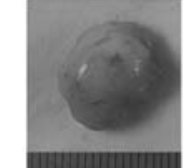

D

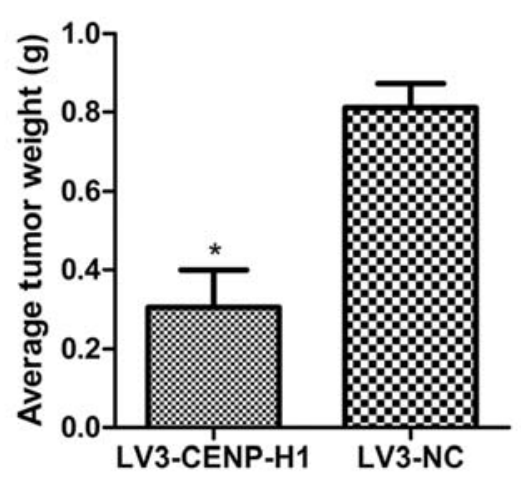

Figure 6. CENP-H knockdown inhibits tumor growth in Hep3B xenograft models. (A) LV3-NC subcutaneous tumor-bearing mice and explanted tumors. (B) LV3-CENP-H1 subcutaneous tumor-bearing mice and explanted tumors. Subcutaneous tumors in nude mice were monitored over time. (C) Average tumor volume over time. (D) Average tumor weight. Values are expressed as the mean \pm standard error; ${ }^{*} \mathrm{P}<0.05$ vs. the LV3-NC cells. CENP-H, centromere protein-H.

associated with patient prognosis (15). In the present study we further confirmed that the expression levels of CENP-H were higher in the HCC cell lines than that in the L02 immortalized human liver cells. CENP-H knockdown inhibited the proliferation of Hep3B cells and induced their apoptosis in vitro. Furthermore, tumor growth in the Hep3B xenograft model was inhibited after CENP-H knockdown. The role of CENP-H in HCC growth may be mediated through the mitochondrial apoptotic signaling pathway. All of these results confirmed that CENP-H may play a key role in the regulation of HCC growth.

Uncontrolled proliferation is an obvious characteristic of cancers, and tumor growth can be inhibited by controlling the proliferation of tumor cells. Orthaus et al (17) reported that depletion of CENP-H resulted in the decreased number of living cells and increased cell death in human HEp-2 cells by RNAi knockdown of CENP-H. Compared with the control cells, the population doubling time was shorter and the colony formation ability was decreased in Tca8113/CENP-H RNAi cells, which highlighted the compromised viability caused by the downregulation of CENP-H (18). Consistent with these studies $(12,17,18)$, our MTT and colony formation data also revealed that shRNA-mediated CENP-H knockdown caused Hep3B proliferation inhibition. Similarly to the in vitro experiments,LV3-CENP-H1 cells exhibited decreased tumorigenicity in nude mice in vivo. $\mathrm{Ki}-67$, a proliferation marker of many malignancies including HCC (19), was found to be expressed at lower levels in the LV3-CENP-H1 subcutaneous xenograft tumors. These results collectively demonstrated that CENP-H is closely associated with the proliferation of $\mathrm{HCC} \mathrm{Hep3B} \mathrm{cells.}$

In the present study, we also analyzed the effects of CENP-H knockdown on the apoptosis of Hep3B cells by flow cytometry and TEM. Both results revealed that CENP-H knockdown increased apoptosis in the Hep3B cells. Moreover, the cleaved caspase-3 protein expression increased after CENP-H knockdown based on western blotting. Survivin, a member of the inhibitor of apoptosis protein family, has been implicated as a protector against cell apoptosis; the aberrant high expression of this factor in cancers is closely correlated with advanced disease and poor overall survival $(20,21)$. According to previous studies, CENP-H siRNA decreases the protein expression of survivin, indicating apoptosis induction $(12,18)$. Wang et al (22) found that siRNA knockdown of CENP-H mRNA promoted FaDu cell apoptosis. All of these results are consistent with our findings.

Considering the changes in mitochondria observed under TEM, the factors involved in the mitochondrial apoptotic pathway were examined by western blotting and real-time PCR (23). The results demonstrated that CENP-H knockdown not only induced the activation of caspase- 3 in Hep3B cells with increased protein expression of cleaved caspase- 3 but also resulted in a Bax/Bcl-2 imbalance with a significant increase of Bax expression and an obvious decrease in Bcl-2 expression at both the mRNA and protein levels. All of these 

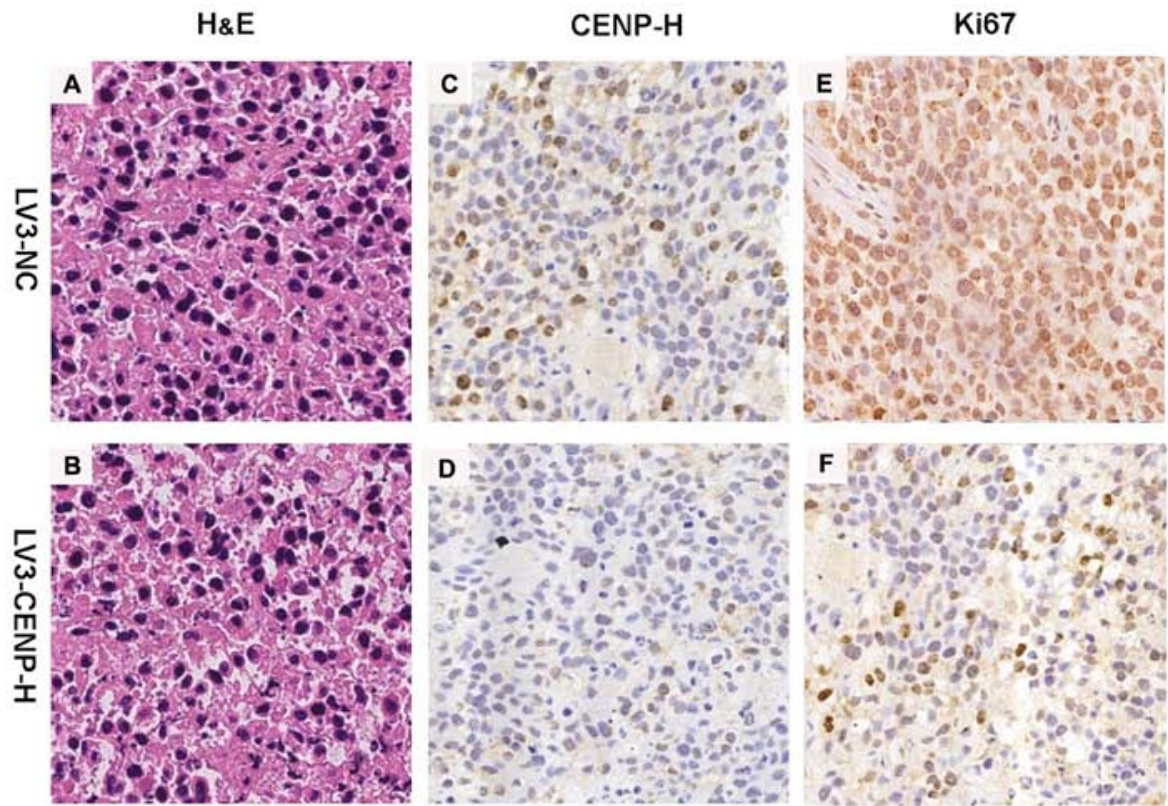

Cleaved Caspase-3

Bax

$\mathrm{Bcl}-2$
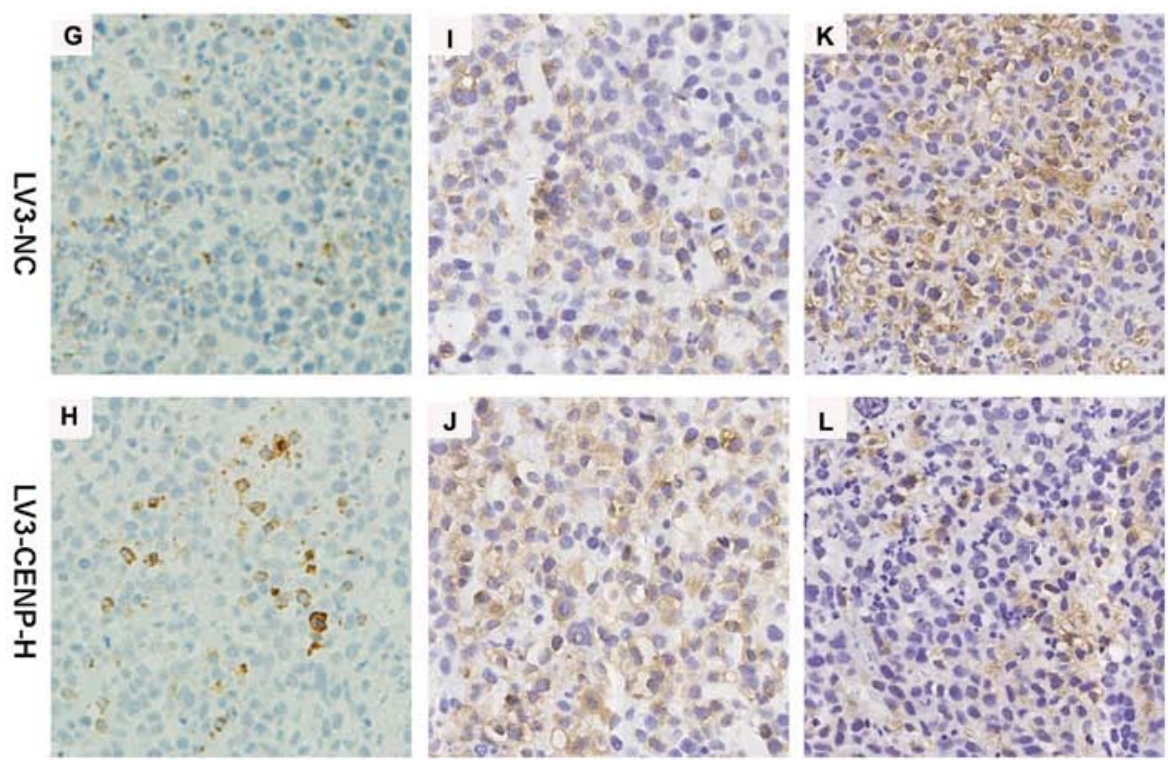

Figure 7 Representative immunohistochemical results in xenograft models. (A and B) H\&E staining. (C and D) CENP-H staining. (E and F) Ki-67 staining. $(\mathrm{G}$ and $\mathrm{H}$ ) Cleaved caspase-3 staining. (I and J) Bax staining. (K and L) Bcl-2 staining in xenograft models. CENP-H, centromere protein-H.

results were further ascertained in subcutaneous xenograft models. CENP-A, another inner centromere protein, has been shown to be upregulated in HCC. CENP-A siRNA induced apoptosis in HepG2 cells with increased expression of Bax and decreased expression of Bcl-2 (24), which was consistent with our results. Bax and Bcl-2 are two of the most important members of the Bcl-2 family; anti-apoptotic $\mathrm{Bcl}-2$ protein prevents apoptosis by inhibiting the activation of pro-apoptotic Bax protein (25). Increased $\mathrm{Bax} / \mathrm{Bcl}-2$ ratio results in the release of cytochrome $c$, which cooperates with cytosolic factors to activate the caspases (25-27). Once the executioner caspase- 3 is activated, apoptosis is inevitable $(28,29)$. In the present study, we found that apoptosis was accompanied by a Bax/Bcl-2 imbalance and activation of caspase-3 at both the cellular and tissue levels after CENP-H knockdown. These results collectively suggested that targeting CENP-H induced HCC cell apoptosis through the mitochondrial apoptotic pathway.

In conclusion, CENP-H was upregulated in HCC cells, and CENP-H knockdown inhibited Hep3B cell proliferation both in vitro and in vivo. The mitochondrial apoptotic pathway mediated by a Bax/Bcl-2 imbalance may be involved in apoptosis induction by CENP-H knockdown. All of these data suggested that CENP-H may be a potential therapeutic target for HCC.

\section{Acknowledgements}

The present study was supported by the International Cooperation Project of Shaanxi Province (2015KW-043). The present study also received support from the National Natural Science Foundation of China (no. 30771895). We thank 
the staff of the Transform Medical Center, Xi'an Jiaotong University for their technical assistance in these studies.

\section{References}

1. Torre LA, Bray F, Siegel RL, Ferlay J, Lortet-Tieulent J and Jemal A: Global cancer statistics, 2012. CA Cancer J Clin 65: 87-108, 2015

2. Chen W, Zheng R, Baade PD, Zhang S, Zeng H, Bray F, Jemal A, Yu XQ and He J: Cancer statistics in China, 2015. CA Cancer J Clin 66: 115-132, 2016

3. Siegel RL, Miller KD and Jemal A: Cancer statistics, 2015. CA Cancer J Clin 65: 5-29, 2015

4. Zender L, Villanueva A, Tovar V, Sia D, Chiang DY and Llovet JM: Cancer gene discovery in hepatocellular carcinoma. J Hepatol 52: 921-929, 2010.

5. Schvartzman JM, Sotillo R and Benezra R: Mitotic chromosomal instability and cancer: Mouse modelling of the human disease. Nat Rev Cancer 10: 102-115, 2010.

6. Westhorpe FG and Straight AF: Functions of the centromere and kinetochore in chromosome segregation. Curr Opin Cell Biol 25 334-340, 2013

7. Pfau SJ and Amon A: Chromosomal instability and aneuploidy in cancer: From yeast to man. EMBO Rep 13: 515-527, 2012.

8. Fukagawa T, Mikami Y, Nishihashi A, Regnier V, Haraguchi T, Hiraoka Y, Sugata N, Todokoro K, Brown W and Ikemura T: $\mathrm{CENP}-\mathrm{H}$, a constitutive centromere component, is required for centromere targeting of CENP-C in vertebrate cells. EMBO J 20 : 4603-4617, 2001.

9. Tomonaga T, Matsushita K, Ishibashi M, Nezu M, Shimada H, Ochiai T, Yoda $\mathrm{K}$ and Nomura F: Centromere protein $\mathrm{H}$ is up-regulated in primary human colorectal cancer and its overexpression induces aneuploidy. Cancer Res 65: 4683-4689, 2005

10. Guo XZ, Zhang G, Wang JY, Liu WL, Wang F, Dong JQ, $\mathrm{Xu} \mathrm{LH}$, Cao JY, Song LB and Zeng MS: Prognostic relevance of Centromere protein $\mathrm{H}$ expression in esophageal carcinoma. BMC Cancer 8: 233, 2008.

11. Quan T, He B, Liu T, Li W, Wu S, Jiang Q, Liu W, Liu H and $\mathrm{Xu} \mathrm{X}$ : Role of centromere protein $\mathrm{H}$ in human gastric cancer cell proliferation. Nan Fang Yi Ke Da Xue Xue Bao 32: 265-269, 2012 (In Chinese).

12. He WL, Li YH, Yang DJ, Song W, Chen XL, Liu FK, Wang Z, $\mathrm{Li}$ W, Chen W, Chen CY, et al: Combined evaluation of centromere protein $\mathrm{H}$ and $\mathrm{Ki}-67$ as prognostic biomarker for patients with gastric carcinoma. Eur J Surg Oncol 39: 141-149, 2013.

13. Liao WT, Feng Y, Li ML, Liu GL, Li MZ, Zeng MS and Song LB Overexpression of centromere protein $\mathrm{H}$ is significantly associated with breast cancer progression and overall patient survival. Chin J Cancer 30: 627-637, 2011.

14. Weng MY, Li L, Hong SJ and Feng SY: Clinical significance of CENP-H expression in uterine cervical cancer. Cancer Biol Med 9: 192-196, 2012.
15. Lu G, Shan T, He S, Ren M, Zhu M, Hu Y, Lu X and Zhang D: Overexpression of CENP-H as a novel prognostic biomarker for human hepatocellular carcinoma progression and patient survival. Oncol Rep 30: 2238-2244, 2013.

16. Forner A, Llovet JM and Bruix J: Hepatocellular carcinoma. Lancet 379: 1245-1255, 2012

17. Orthaus S, Ohndorf S and Diekmann S: RNAi knockdown of human kinetochore protein CENP-H. Biochem Biophys Res Commun 348: 36-46, 2006.

18. Liao WT, Yu CP, Wu DH, Zhang L, Xu LH, Weng GX, Zeng MS, Song LB and Li JS: Upregulation of CENP-H in tongue cancer correlates with poor prognosis and progression. J Exp Clin Cancer Res 28: 74, 2009.

19. Luo Y, Ren F, Liu Y, Shi Z, Tan Z, Xiong H, Dang Y and Chen G: Clinicopathological and prognostic significance of high Ki-67 labeling index in hepatocellular carcinoma patients: A metaanalysis. Int J Clin Exp Med 8: 10235-10247, 2015.

20. Athanasoula KC, Gogas H, Polonifi K, Vaiopoulos AG, Polyzos A and Mantzourani M: Survivin beyond physiology: Orchestration of multistep carcinogenesis and therapeutic potentials. Cancer Lett 347: 175-182, 2014.

21. Andersen MH, Svane IM, Becker JC and Straten PT: The universal character of the tumor-associated antigen survivin. Clin Cancer Res 13: 5991-5994, 2007.

22. Wang JX, Zhang YY, Yu XM, Jin T and Pan XL: Role of centromere protein $\mathrm{H}$ and $\mathrm{Ki} 67$ in relapse-free survival of patients after primary surgery for hypopharyngeal cancer. Asian Pac J Cancer Prev 13: 821-825, 2012.

23. Lakhani SA, Masud A, Kuida K, Porter GA Jr, Booth CJ, Mehal WZ, Inayat I and Flavell RA: Caspases 3 and 7: Key mediators of mitochondrial events of apoptosis. Science 311: 847-851, 2006.

24. Li Y, Zhu Z, Zhang S, Yu D, Yu H, Liu L, Cao X, Wang L, Gao H and Zhu M: ShRNA-targeted centromere protein A inhibits hepatocellular carcinoma growth. PLoS One 6: e17794, 2011.

25. Lindsay J, Esposti MD and Gilmore AP: Bcl-2 proteins and mitochondria - specificity in membrane targeting for death. Biochim Biophys Acta 1813: 532-539, 2011.

26. Autret A and Martin SJ: Emerging role for members of the Bcl-2 family in mitochondrial morphogenesis. Mol Cell 36: 355-363, 2009.

27. Howells CC, Baumann WT, Samuels DC and Finkielstein CV: The Bcl-2-associated death promoter (BAD) lowers the threshold at which the Bcl-2-interacting domain death agonist (BID) triggers mitochondria disintegration. J Theor Biol 271: 114-123, 2011.

28. Taylor RC, Cullen SP and Martin SJ: Apoptosis: Controlled demolition at the cellular level. Nat Rev Mol Cell Biol 9: 231-241, 2008.

29. Brancolini C, Lazarevic D, Rodriguez J and Schneider C: Dismantling cell-cell contacts during apoptosis is coupled to a caspase-dependent proteolytic cleavage of beta-catenin. J Cell Biol 139: 759-771, 1997. 